\title{
Gait Biomechanics Following Taping and Bracing in Patients With Chronic Ankle Instability: A Critically Appraised Topic
}

\author{
Kimmery Migel and Erik Wikstrom
}

\begin{abstract}
Clinical Scenario: Approximately 30\% of all first-time patients with LAS develop chronic ankle instability (CAI). CAIassociated impairments are thought to contribute to aberrant gait biomechanics, which increase the risk of subsequent ankle sprains and the development of posttraumatic osteoarthritis. Alternative modalities should be considered to improve gait biomechanics as impairment-based rehabilitation does not impact gait. Taping and bracing have been shown to reduce the risk of recurrent ankle sprains; however, their effects on CAI-associated gait biomechanics remain unknown. Clinical Question: Do ankle taping and bracing modify gait biomechanics in those with CAI? Summary of Key Findings: Three casecontrol studies assessed taping and bracing applications including kinesiotape, athletic tape, a flexible brace, and a semirigid brace. Kinesiotape decreased excessive inversion in early stance, whereas athletic taping decreased excessive inversion and plantar flexion in the swing phase and limited tibial external rotation in terminal stance. The flexible and semirigid brace increased dorsiflexion range of motion, and the semirigid brace limited plantar flexion range of motion at toe-off. Clinical Bottom Line: Taping and bracing acutely alter gait biomechanics in those with CAI. Strength of Recommendation: There is limited quality evidence (grade B) that taping and bracing can immediately alter gait biomechanics in patients with CAI.
\end{abstract}

Keywords: biomechanics, gait, rehabilitation, ankle

\section{Clinical Scenario}

Approximately 3.1 million lateral ankle sprains (LASs) are diagnosed each year. ${ }^{1}$ These seemingly innocuous injuries are significant as about $30 \%$ of all first-time patients with LAS develop chronic ankle instability (CAI). ${ }^{2} \mathrm{CAI}$ is a musculoskeletal condition characterized by recurrent sprains, repeated episodes of giving way, persistent pain, and decreased self-reported function. ${ }^{2}$ Individuals with CAI also demonstrate sensorimotor dysfunction, ligamentous laxity, limited range of motion, decreased strength, and decreased neuromuscular control. ${ }^{2}$ These impairments are thought to contribute to aberrant gait biomechanics observed in those with CAI. More specifically, those with CAI demonstrate excessive inversion at heel strike and throughout stance as well as excessive inversion and plantar flexion in the swing phase of gait. $^{2}$ These impairments increase the risk of subsequent $\mathrm{LAS}^{3}$ and can alter talar-loading patterns, leading to the development of posttraumatic osteoarthritis. ${ }^{2}$ Gait adaptations have been found to persist despite participation in traditional rehabilitation program focusing on strength, range of motion, and neuromuscular control. ${ }^{2}$ Therefore, alternative modalities should be considered to help improve gait biomechanics until effective rehabilitation programs can be developed. Taping and bracing are commonly used to supplement impairment-based rehabilitation programs for individuals with CAI and have been shown to reduce the risk of recurrent ankle sprains. ${ }^{4}$ However, their effects on CAI frontal and sagittal plane gait kinematics remain unknown.

\section{Focused Clinical Question}

Do taping and bracing of the ankle improve frontal and sagittal plane ankle joint gait kinematics in individuals with CAI?

\section{Summary of Search, "Best Evidence" Appraised, and Key Findings}

- The literature was searched for studies of level 3 evidence or higher (case-control studies through systematic reviews) that investigated the effect of taping and bracing on level ground frontal and sagittal plane ankle joint gait kinematics in those with CAI.

- Three case-control studies ${ }^{5-7}$ met our inclusion criteria, and all had high validity (PEDro) scores of 7/10 or higher.

- All studies assessed a taping or bracing application common to clinical practice. Two studies ${ }^{5,7}$ investigated taping techniques (kinesiotape $^{7}$ and athletic tape ${ }^{5,7}$ ) compared with a nontaped, control condition. The remaining study ${ }^{6}$ addressed a flexible and semirigid brace compared with a nonbraced, control condition.

- Kinesiotape was found to decrease excessive inversion in early stance in individuals with CAI. ${ }^{5,7}$ Athletic taping decreased excessive inversion and plantar flexion throughout the swing phase. ${ }^{7}$

- At heel strike, both a flexible and a semirigid brace increased dorsiflexion range of motion, whereas at toe-off, only a semirigid brace limited plantar flexion range of motion. ${ }^{6}$

\section{Clinical Bottom Line}

The authors are with the University of North Carolina at Chapel Hill, Chapel Hill, NC. Migel (kmigel@live.unc.edu) is corresponding author.
Evidence indicates that taping and bracing acutely improve frontal and sagittal plane ankle joint gait kinematics in those with CAI. 


\section{Strength of Recommendation}

There is limited quality evidence (grade B) that taping and bracing can immediately improve frontal and sagittal plane ankle joint gait kinematics in patients with CAI.

\section{Search Strategy}

\section{Terms Used to Guide Search Strategy}

Patient/Client group: chronic ankle instability or ankle instability or functional ankle instability or mechanical ankle instability or lateral ankle sprain

Intervention: tap* or brac*

Comparison: NA

Qutcomes: gait or walking or biomechanics or kinematics or kinetics

\section{Sources of Evidence Searched}

- PubMed, MEDLINE, and CINAHL from inception to January 11, 2019. Reference lists of identified articles were also reviewed.

\section{Inclusion and Exclusion Criteria}

\section{Inclusion Criteria}

- Studies that investigated the preeffects to posteffects of taping or bracing on frontal and sagittal plane ankle joint gait kinematics in patients with CAI.

- Level 3 evidence or higher.

- Limited to English language.

\section{Exclusion Criteria}

- Studies that did not use participants with CAI.

- Studies using any intervention strategy other than taping and bracing.

- Studies that did not assess frontal and sagittal plane ankle joint gait kinematics.

- Studies where the full text was not available in English.

\section{Results of the Search}

Three studies met our inclusion criteria and are presented in Table 1.

\section{Best Evidence}

The studies selected for inclusion in this critically appraised topic are listed in Table 2. The included studies met all inclusion and exclusion criteria.

Table 1 Summary of Study Designs

\begin{tabular}{|c|c|c|}
\hline Author & Study design & Level of evidence \\
\hline Chinn et al $^{5}$ & Case-control, crossover & $3 b$ \\
\hline Spaulding et $\mathrm{al}^{6}$ & Case-control, crossover & $3 b$ \\
\hline Yen et $\mathrm{al}^{7}$ & Case-control, crossover & $3 b$ \\
\hline
\end{tabular}

\section{Implications for Practice, Education, and Future Research}

The studies included in this critically appraised topic ${ }^{5-7}$ support the use of taping and bracing to change ankle joint gait kinematics in individuals with CAI. Athletic tape decreased excessive plantar flexion in the swing phase, ${ }^{7}$ and kinesiotape decreased excessive inversion in early stance. ${ }^{5,7}$ At heel strike, the flexible brace and the semirigid brace both increased dorsiflexion range of motion compared with a nonbraced condition, and at toe-off, the semirigid brace limited plantar flexion. ${ }^{6}$

Individuals with CAI are known to have excessive supination during the stance phase, and excessive plantar flexion and inversion in the swing phase, ${ }^{2}$ which may increase vulnerability to subsequent LAS. Given these aberrant biomechanics, the application of taping and bracing can be used to acutely alter gait in those with CAI to create a biomechanical profile closer to that of healthy controls. Sagittal plane ankle joint kinematics improved across all studies, ${ }^{5-7}$ whereas frontal plane ankle joint kinematics were only impacted by kinesiotape, ${ }^{7}$ not athletic tape or braces. ${ }^{5-7}$ However, it is important to note that semirigid braces may negatively impact push-off force as they create the sizable limitation in plantar flexion range of motion at toe-off. ${ }^{6}$

All 3 studies $^{5-7}$ suggest immediate positive effects of taping and bracing during a single walking session. However, there remain a number of criticisms surrounding the integrity of tape over time due to a potential loosening effect. Larsen ${ }^{8}$ found that following 20 minutes of athletic activity, almost all gains due to a tape application were lost. However, Hubbard and Cordova ${ }^{9}$ noted that following a 30-minute multidirectional running protocol, athletic tape still provided mechanical support. Similarly, Delahunt et $\mathrm{al}^{10}$ found no changes in drop-landing kinematics after 25 minutes of continuous hopping and sport-specific drills while taped. Cumulatively, the literature suggests that while tape appears to loosen over time, benefits do remain. However, the effects of tape over a longer period such as an entire sporting event remain unknown.

Although taping and bracing were observed to alter frontal and sagittal plane ankle joint kinematics in those with CAI, it remains unknown if the improvements are due to restricted range of motion or increased sensory stimulation. The results of Larsen ${ }^{8}$ and Hubbard and Cordova ${ }^{9}$ suggest that there may be a mechanical mechanism; however, these results do not explain the improved gait biomechanics following application of kinesiotape. Being elastic in nature, kinesiotape does not provide a mechanical restriction, and therefore, it does not limit joint laxity or range of motion, suggesting that tape may also work, at least in part, through increased sensory stimulation. Those with CAI are known to have deficits in sensorimotor control, and therefore postural control. ${ }^{2}$ However, postural control improves up to 48 hours after kinesiotape application, ${ }^{11}$ providing additional evidence that kinesiotape tape may work through sensory input modulation. Future research must explore these potential mechanisms and the long-term effects (eg, accommodation) of taping and bracing on gait biomechanics.

Historically, taping and bracing have been used in the acute phase of healing to protect injured tissues. Long-term taping and bracing are anecdotally criticized for potentially hindering muscle activation and failing to promote motor learning (relative to therapeutic exercise), despite a lack of evidence to support such criticism. However, it is unlikely that an individual can be taped/braced in all situations for a variety of reasons (eg, logistical constraints, financial restrictions, skin breakdown). Therefore, individuals with CAI would be at an increased risk of recurrent ankle sprains during 


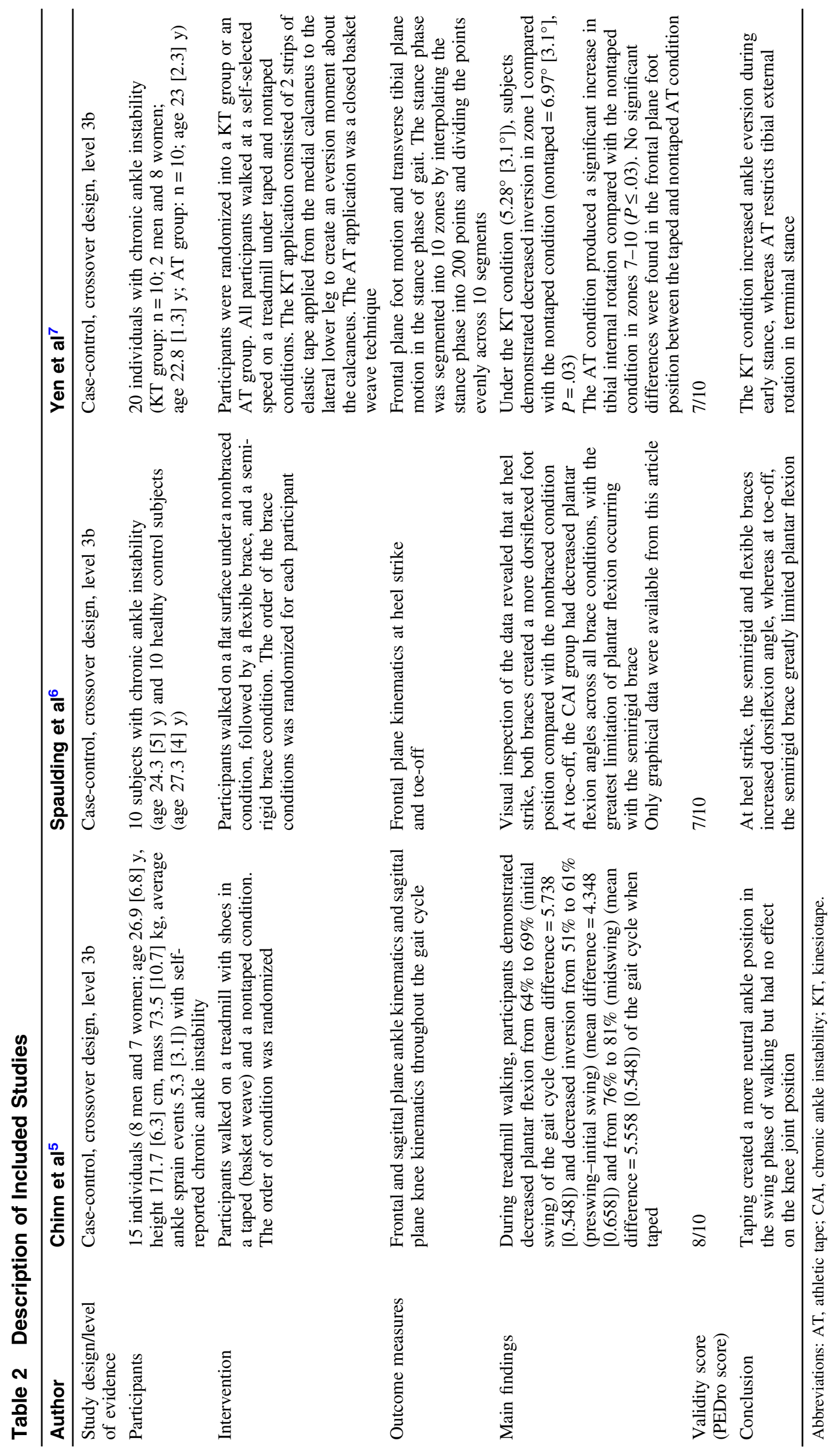


these unprotected exposures. Based on the current evidence, we recommend that taping and bracing be used early during a comprehensive rehabilitation program to help promote proper gait biomechanics and reduce ankle sprain risk in those with CAI.

\section{Acknowledgment}

There are no conflicts of interest to declare.

\section{References}

1. Waterman BR, Owens BD, Davey S, Zacchilli MA, Belmont PJ Jr. The epidemiology of ankle sprains in the United States. J Bone Joint Surg Am. 2010;92(13):2279-2284. PubMed ID: 20926721 doi:10. 2106/JBJS.I.01537

2. Gribble PA, Bleakley CM, Caulfield BM, et al. Evidence review for the 2016 International Ankle Consortium consensus statement on the prevalence, impact and long-term consequences of lateral ankle sprains. Br J Sports Med. 2016;50(24):1496-1505. PubMed ID: 27259753 doi:10.1136/bjsports-2016-096189

3. Willems T, Witvrouw E, Delbaere K, De Cock A, De Clercq D. Relationship between gait biomechanics and inversion sprains: a prospective study of risk factors. Gait Posture. 2005;21(4):379-387. PubMed ID: 15886127 doi:10.1016/j.gaitpost.2004.04.002

4. Janssen KW, van Mechelen W, Verhagen EA. Bracing superior to neuromuscular training for the prevention of self-reported recurrent ankle sprains: a three-arm randomised controlled trial. Br J Sports
Med. 2014;48(16):1235-1239. PubMed ID: 24398222 doi:10.1136/ bjsports-2013-092947

5. Chinn L, Dicharry J, Hart JM, Saliba S, Wilder R, Hertel J. Gait kinematics after taping in participants with chronic ankle instability. J Athl Train. 2014;49(3):322-330. PubMed ID: 24840583 doi:10. 4085/1062-6050-49.3.08

6. Spaulding SJ, Livingston LA, Hartsell HD. The influence of external orthotic support on the adaptive gait characteristics of individuals with chronically unstable ankles. Gait Posture. 2003;17(2):152-158. PubMed ID: 12633776

7. Yen SC, Folmar E, Friend KA, Wang YC, Chui KK. Effects of kinesiotaping and athletic taping on ankle kinematics during walking in individuals with chronic ankle instability: a pilot study. Gait Posture. 2018;66:118-123. PubMed ID: 30176379 doi:10.1016/j. gaitpost.2018.08.034

8. Larsen E. Taping the ankle for chronic instability. Acta Orthop Scand. 1984;55(5):551-553. PubMed ID: 6507080

9. Hubbard TJ, Cordova M. Effect of ankle taping on mechanical laxity in chronic ankle instability. Foot Ankle Int. 2010;31(6):499-504. PubMed ID: 20557815 doi:10.3113/FAI.2010.0499

10. Delahunt E, O'Driscoll J, Moran K. Effects of taping and exercise on ankle joint movement in subjects with chronic ankle instability: a preliminary investigation. Arch Phys Med Rehabil. 2009;90(8):14181422. PubMed ID: 19651278 doi:10.1016/j.apmr.2009.01.024

11. Jackson K, Simon JE, Docherty CL. Extended use of kinesiology tape and balance in participants with chronic ankle instability. $J$ Athl Train. 2016;51(1):16-21. PubMed ID: 26752273 doi:10.4085/10626050-51.2.03 